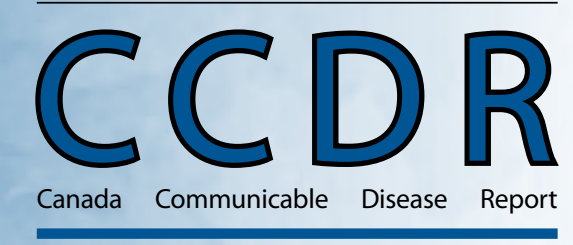

ISSN 1188-4169

Volume: 3652

January 2010

\title{
Supplement
}

\section{ADVICE FOR CONSIDERATION OF QUADRIVALENT (A, C, Y, W135) MENINGOCOCCAL CONJUGATE VACCINE, FOR USE BY PROVINCES AND TERRITORIES}

Canadian Immunization Committee 


\section{Acknowledgment}

The Centre for Immunization and Respiratory Infections of the Public Health Agency of Canada, through a contract with Dr. Philippe De Wals from the Public Health Research Unit, Quebec University Hospital Research Centre, requested an evaluation of the quadrivalent meningococcal vaccine using the Erickson, De Wals and Farand Analytical Framework. This report is primarily based on the evaluation provided by Dr. De Wals and represents available epidemiological and economical information up to Sept.2007. The information on the burden of disease and the vaccine characteristics has been updated in a statement from the National Advisory Committee on Immunization (NACI) in 2009.

The scientific review, critical analysis and expert opinion presented by Dr. De Wals in this report are acknowledged.

\section{Suggested citation:}

Canadian Immunization Committee and Public Health Agency of Canada

Advice for Consideration of Quadrivalent (A, C, Y, W135) Meningococcal Conjugate Vaccine, for Use by Provinces and Territories CCDR 2009

This publication can also be accessed electronically via Internet using a Web browser at: http://www.phac-aspc.gc.ca

() Her Majesty the Queen in Right of Canada, 2009 


\section{ADVICE FOR CONSIDERATION OF QUADRIVALENT (A, C, Y, W135) MENINGOCOCCAL CONJUGATE VACCINE, FOR USE BY PROVINCES AND TERRITORIES}




\section{Title of Contents}

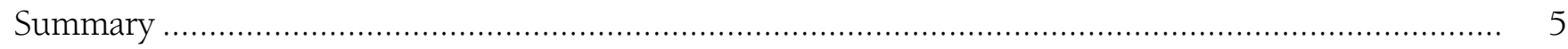

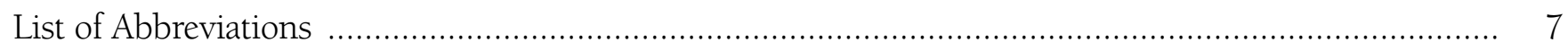

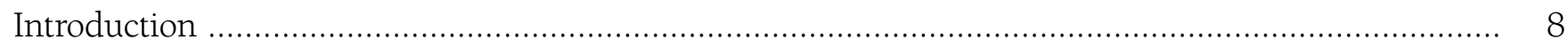

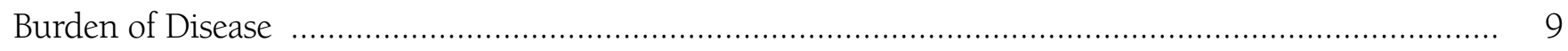

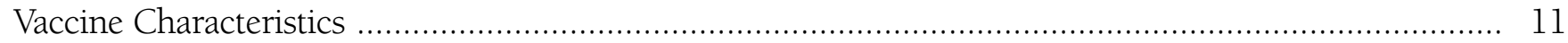

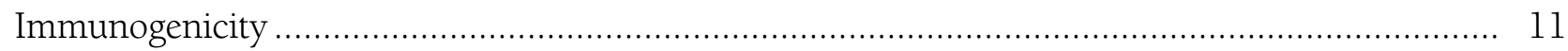

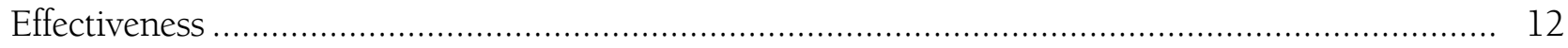

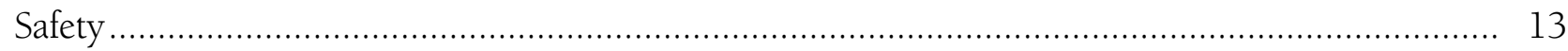

Concomitant Vaccine Administration ....................................................................... 14

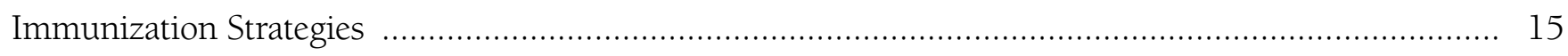

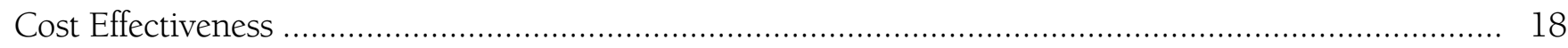

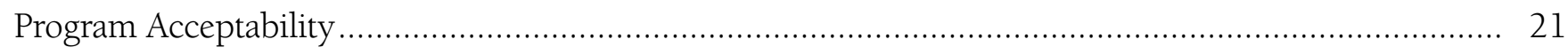

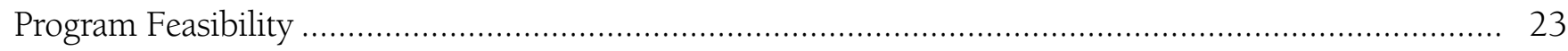

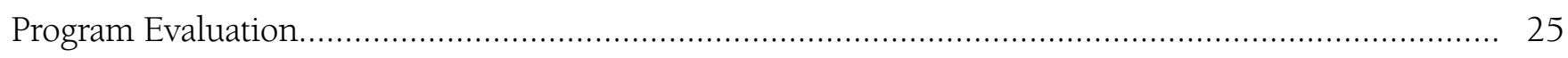

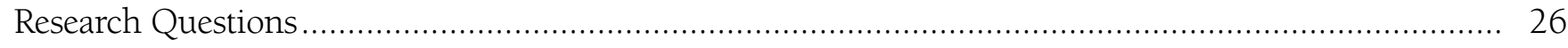

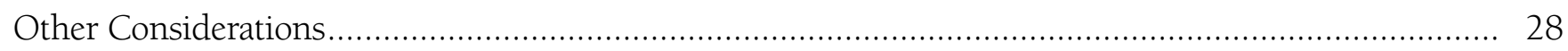

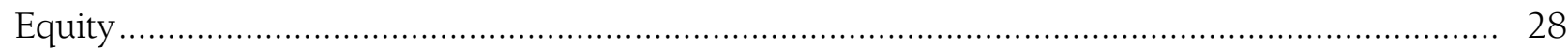

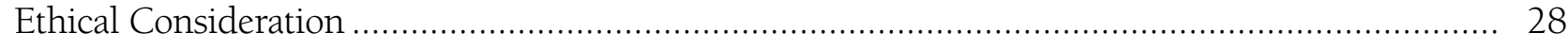

CIC Recommendation for Use of Meningococcal Vaccines .............................................. 28

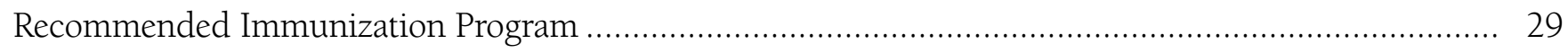

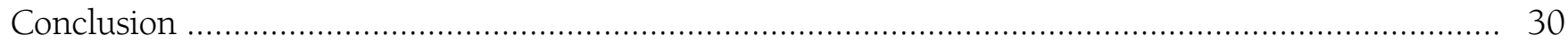

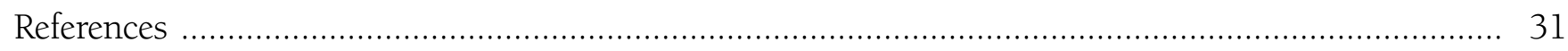




\section{List of Figures}

Figure 1: Age-specific risk of vaccine-preventable invasive meningococcal disease in Canada

(De Wals et al., 2007)

\section{List of Tables}

Table 1: Recommendations regarding immunization schedule against invasive serogroup C meningococcal disease in Canadian provinces and territories (PHAC 2007/12/28)

Table 2: Estimated program cost to the health system according to different population coverage of teenagers with a quadrivalent meningococcal conjugate vaccine in Canada

Table 3: Cost-effectiveness of different immunization strategies using the serogroup C meningococcal conjugate vaccine (MenC-C) or quadrivalent meningococcal conjugate vaccine (Men4-DT) in a population of one million in Canada, in base model scenario (De Wals et al., 2007).... 20

Table 4: Vaccination programs for 9 to 16 year-olds in Canadian provinces and territories (PHAC 2007b). 


\section{Summary}

In 2006, the first quadrivalent meningococcal conjugate vaccine (Menactra ${ }^{\mathrm{TM}}$ ) was authorized for use in Canada. This statement provides the evidence based analysis needed for consideration of this vaccine for immunization program planning by provinces and territories. Provinces and territories are responsible for the delivery of immunization programs and will consider their own circumstances in making decisions about the vaccine based on the critical analysis provided in this report.

Historically, the epidemiology of meningococcal disease in Canada has been characterized by an endemic background and occurrence of large epidemics and smaller outbreaks at irregular intervals. There are marked differences between serogroups, serogroup B strains causing the majority of sporadic cases and virulent serogroup C clones causing most of the outbreaks observed during the last two decades. To date, it has not been possible to reliably predict the epidemiology of meningococcal disease, therefore the occurrence of new epidemics or outbreaks caused by virulent clones of any serotype cannot be excluded in Canada.

In all existing trials in persons aged 2 years or more, the short-term immunologic response to Menactra $^{\mathrm{TM}}$ was non-inferior to the response to the quadrivalent polysaccharide vaccine.

Menactra ${ }^{\mathrm{TM}}$ generated antibodies having better functional characteristics and longer persistence, while inducing a strong anamnestic response following a booster dose without hyporesponsiveness. Thus, Menactra ${ }^{\mathrm{TM}}$ should be preferred to the quadrivalent polysaccharide vaccine for all indications of a quadrivalent or a serogroup A, Y or W135 meningococcal vaccine those aged 2 years and older. The field effectiveness of monovalent serogroup $C$ conjugate vaccines has been evaluated in large postmarketing epidemiological studies. Currently, there are no data on the clinical effectiveness of Menactra ${ }^{\mathrm{TM}}$. There has been no immunologic study providing a head-to-head comparison of Menactra $^{\mathrm{TM}}$ with field tested serogroup C conjugate vaccines containing either the CRM197 carrier protein derived from the diphtheria toxoid (Meningitec $^{\mathrm{TM}}$, and Menjugate ${ }^{\mathrm{TM}}$ ) or the tetanus toxoid (Neis Vac-C ${ }^{\mathrm{TM}}$ ). Although the immune response of Menactra ${ }^{\mathrm{TM}}$ was compared to the response of the polysaccharide vaccine, and was non-inferior for serogroup $C$, it is established that the polysaccharide vaccine provides a low level of protection of short duration for this serogroup in children. For these reasons, until the results of new studies are available, monovalent serogroup $\mathrm{C}$ conjugate vaccines should be preferred for all indications of vaccination against serogroup C meningococcal disease, especially for children. While the effectiveness of polysaccharide vaccines to prevent serogroup A and C IMD has been demonstrated in experimental and observational studies in adolescents and adults, immunologic studies have shown that MenactraTM (Men4-DT) is a more potent vaccine. For this reason, Men4-DT could be considered for routine immunization of adolescents, including those already immunized with a monovalent serogroup $\mathrm{C}$ conjugate vaccine at a young age.

The experience of administration of Menactra ${ }^{\mathrm{TM}}$ in nine clinical trials involving more than 10000 subjects is very reassuring and adverse events were not substantially different than those reported in the control groups vaccinated with a quadrivalent polysaccharide vaccine. In the US, Menactra ${ }^{\mathrm{TM}}$ is widely used for vaccinating adolescents and college students. As of February 25, 2008, more than 15 million doses of Menactra $^{\mathrm{TM}}$ have been distributed, and the 
Vaccine Adverse Event Reporting Systems (VAERS) has received 26 confirmed case reports of GBS within 6 weeks of receipt of Menactra ${ }^{\mathrm{TM}}$ meningococcal vaccination. A causal relationship has not been established, but the existence of a risk of very small magnitude cannot be excluded. The preferred indication for Menactra ${ }^{\mathrm{TM}}$ in Canada is for vaccination of adolescents at the age of 12 years, just before the increase in incidence meningococcal disease that occurs at the age of 13 years. For adolescents not previously vaccinated, this would be a primary vaccination, and for those previously vaccinated with a monovalent serogroup $C$ conjugate vaccine at a young age, this would be a booster dose for serogroup $C$ and a primary vaccination for serogroups $\mathrm{A}, \mathrm{Y}$ and W135. Vaccination of an important proportion of adolescents could significantly reduce transmission of pathogenic strains in the entire population, as the prevalence of carriage of $\mathrm{N}$. meningitidis is maximal in this age group.

Using a vaccine purchase price of $\$ 70$ per Menactra $^{\mathrm{TM}}$ dose, annual program costs for adolescents would range between $\$ 19$ and $\$ 29$ million in Canada. A simulation model was developed for assessing both the direct and indirect effects of a booster dose at 12 years of age with either a monovalent $C$ or a quadrivalent
ACYW135 meningococcal conjugate vaccine in a cohort of Canadians immunized at 12 months with a monovalent $C$ conjugate vaccine ( $\$ 23$ per dose). Revaccination at 12 years using the serogroup $C$ vaccine would reduce the burden of disease by $55 \%$ at no marginal cost (a minor saving is predicted). Using Menactra ${ }^{\mathrm{TM}}$ for the booster dose would result in a disease reduction of $78 \%$ for a marginal cost of $\$ 31000$ per QALY gained compared to one dose of serogroup $C$ vaccine at 12 months. Comparing Menactra ${ }^{\mathrm{TM}}$ with serogroup $C$ vaccine as a booster dose, the incremental cost-effectiveness ratio would be $\$ 113000$ per QALY.

Meningococcal disease is amongst the most feared conditions, and the inclusion of a new vaccine in the regular immunization schedule of adolescents would be well received by the majority of the population and health care professionals.

Ideally, meningococcal vaccination during adolescence should be performed at 12 years of age, via a school-based program (Grade 7) and combined with other vaccinations. The decision to use Menactra ${ }^{\mathrm{TM}}$ or a monovalent serogroup C product is an issue of provincial/territorial priorities in resource allocation. 


\section{List of Abbreviations}

IMD: invasive meningococcal disease

Men4-DT: quadrivalent (serogroup A, C, W135 and Y) polysaccharide diphtheria toxoid protein conjugate meningococcal vaccine (Menactra ${ }^{\mathrm{TM}}$ )

MenC-C: monovalent serogroup C meningococcal conjugate vaccine 


\section{Introduction}

In 2006, a first quadrivalent meningococcal conjugate vaccine (Menactra ${ }^{\mathrm{TM}}$ ) was authorized in Canada (Sanofi Pasteur Limited, 2006) and the National Advisory Committee on Immunization published a statement for the use of this vaccine, focusing on the results of immunogenicity and safety studies, and providing indications and recommendations for its administration (NACI, 2007). The objective of this report is to provide a comprehensive evaluation of the potential usefulness of the quadrivalent meningococcal conjugate vaccine in the Canadian context using the analytical framework as proposed by Erickson et al., (2005) and provide recommendations for the use of Menactra ${ }^{\mathrm{TM}}$ vaccine in publicly funded programs in Canada for prevention of meningococcal invasive disease. 


\section{Burden of Disease}

Historically, the epidemiology of IMD in Canada has been characterized by an endemic background and occurrence of large epidemics and smaller outbreaks at irregular intervals. Epidemiology of IMD during the 1995 to 2004 period was recently reviewed by the National Advisory Committee on Immunization (2007). The overall annual incidence rate ranged between 0.6 and 1.1 per 100,000 , with an average of 244 IMD cases reported, and a case fatality rate of $9 \%$. There were marked differences between serogroups.

Serogroup B accounted for the majority of sporadic cases, and $44 \%$ of cases occurred in children aged less than 5 years (median age of cases $=11$ years), and the case fatality rate was $6 \%$. The majority of cases were caused by meningococci of considerable genetic and phenotypic diversity (Ashton et al., 2001). Currently, there is no vaccine against serogroup B meningococci circulating in Canada or North America (Girard et al., 2006).

During the last 20 years, several outbreaks have been caused by virulent strains of serogroup $C$ characterized by the serotype $2 \mathrm{a}$ antigen and belonging to the electrophoretic type 15 clonal complex (Law et al., 2005). From 1995 to 2004, the average annual number of reported serogroup $\mathrm{C}$ cases was 84 . During this period, a gradual decline in the incidence of serogroup $C$ cases was observed and could, in part, be explained by the mass immunization campaigns and routine vaccination programs implemented in the provinces and territories since 2000. The distribution of serogroup C IMD cases according to age was bimodal with the highest incidence rate around 18 years of age and a second peak around one year of age (median age of cases $=19$ years). IMD caused by epidemic serogroup C clones was particularly severe, with a high proportion of fulminant septicaemia cases, especially in adolescents and young adults, a case fatality rate in the $12-14 \%$ range, and a similar proportion of survivors with permanent physical sequelae (Erickson et al., 1998).

Serogroup Y has been a cause of sporadic cases and the incidence was relatively stable between 1995 and 2004, with an average number of cases of 28 per year. The transient increase in the incidence of serogroup $\mathrm{Y}$ observed in the US during the 1990s and early 2000s (Harrison et al., 2006) was not marked in Canada. Multilocus sequence typing identified two unrelated clonal populations of serogroup Y meningococci belonging to the ST-23 and ST-167 clonal complexes (Tsang et al., 2007). Serogroup Y tended to affect older individuals (median age of cases $=45$ years) and the case fatality rate was $7 \%$.

Serogroup W135 strains have not been frequently isolated in recent years, with an annual average of 9 cases reported. The median age of cases was 19 years and the fatality rate was $8 \%$. No cases related to epidemic strains circulating in African and Arab countries have been identified to date.

In 1940-1943, a nationwide epidemic was caused by serogroup A meningococci in Canada. Although this serogroup still causes large epidemics in Africa and in other parts of the world, it has rarely been isolated in recent years, with between 0 and 2 cases reported annually. To estimate the age-specific risk of vaccinepreventable IMD before the implementation of routine immunization programs in Canada, surveillance data for the 1995-2001 period were used (Deeks et al., 1997; Squires et al., 2000; Squires et al., 2004), adjusting for under-diagnosis when PCR diagnosis was not available ( $10 \%$ of IMD cases) (Lorange et al., 2002), for under- 
reporting (5\% of IMD cases) (Rivest et al., 1999), and for IMD cases of unknown serogroup (yearspecific proportion) (Deeks et al., 1997 ; Squires et al., 2000 ; Squires et al., 2004). As shown in Figure 1, the incidence of vaccine-preventable IMD was dominated by serogroup $C$, and the risk was maximal in the 19-year old group

(2.3/100,000 person-years) and minimal in the 30 to 39 years age group $(0.2 / 100,000$ person-years). In children, a peak was seen around one year of age, low rates in primary school ages and an increase starting at age 13 years. Age-specific mortality rates in Canada in 1990-1992 were used to compute the number of persons at risk in each single year age group of a particular birth cohort (Statistics Canada, 1996). The estimated lifetime risk of vaccine-preventable IMD was 44 cases per 100,000, including 33 cases of serogroup C, 9 cases of serogroup Y, 2 cases of serogroup W135, and less than one case of serogroup A.

Figure 1: Age-specific risk of vaccine-preventable invasive meningococcal disease in Canada (De Wals et al., 2007)

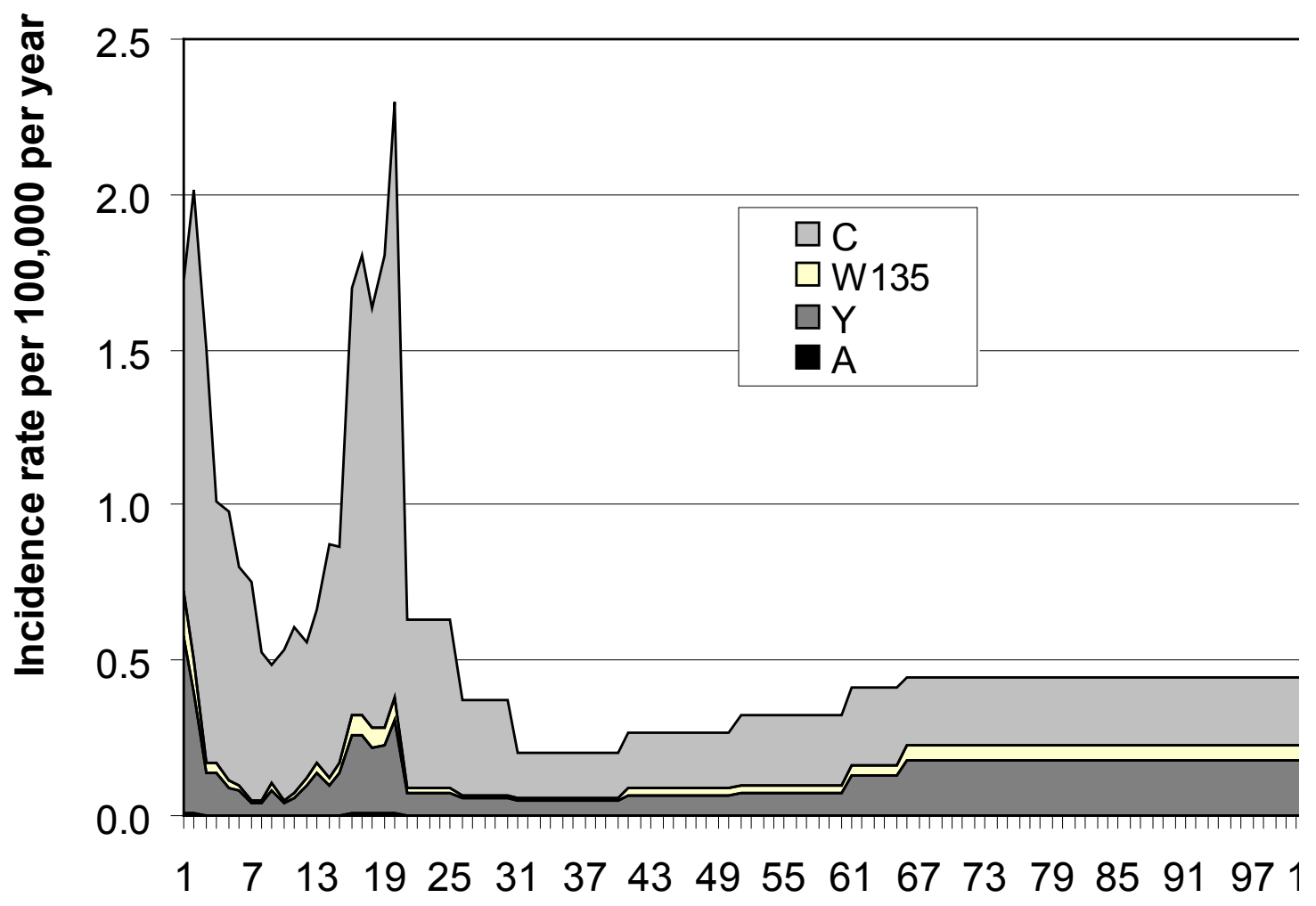

To date, it has not been possible to reliably predict the epidemiology of IMD in any part of the world. In industrialized countries, IMD endemic rates have remained relatively stable over the last century and epidemics or outbreaks have been mainly caused by serogroup A, B and C meningococci. (Rosenstein et al., 2001; Pollard, 2004; Stephens et al., 2007). In the African
Meningitis Belt, epidemics are mainly associated with serogroup A clones and recently, smaller outbreaks have been caused by emerging clones of serogroup W135 and Y. There is potential for importation of these virulent strains, and the occurrence of new epidemics or outbreaks caused by virulent clones of any serotype cannot be excluded in Canada. 


\section{Vaccine Characteristics}

\section{Immunogenicity}

Meningococcal conjugate vaccines, like other polysaccharide-protein conjugate vaccines, elicit a thymus-dependent immunologic response that is already present at birth, and is characterized by the development of memory B cells and by the production of a diversity of immunoglobulin classes and subclasses with high affinity for the capsular polysaccharide antigen (Stein, 1992). Regardless of the type of vaccine, age at vaccine administration and number of doses, serum antibody concentrations decrease with time, while memory is longlasting (De Wals, 2006). The mechanisms of protection provided by meningococcal conjugate vaccines are not entirely understood, but it is known that both mucosal and serum antibodies directed against capsular polysaccharide play a major role through binding of bacterial surface structures and activation of complement leading to phagocytosis or direct bacterial killing (Pollard et al., 2001). Complement-mediated serum bactericidal activity (SBA) measured in vitro is a recognized marker of protection. In studies in the US, there was an inverse correlation between age-specific incidence of meningococcal disease and age-specific prevalence of SBA at the population level (Goldschneider at al., 1969). In a prospective study among military recruits, serogroup C IMD occurred rarely among individuals who had circulating SBA titres of $\geq 4$ (Goldschneider at al., 1969). SBA titres of $\geq 4$ using human complement have been proposed as a marker of protection against serogroup C IMD, and thresholds comprised between $\geq 8$ and $\geq 128$ have been proposed for assays relying on rabbit complement (Andrews et al., 2003; Borrow et al., 2005). These thresholds, however, are not associated with absolute protection and serogroup C IMD cases have been observed in individuals who had pre-existing SBA titres $\geq 4$ using human complement (Goldschneider at al.,
1969). Also, lower titres do not necessarily define susceptibility. In $25 \%$ of sera from unimmunized human adults with SBA titers $<4$ (human complement), high-avidity anticapsular antibodies were present in a concentration insufficient to elicit complement-mediated bacteriolysis in vitro, but sufficient to confer protection in an in vivo bacteraemia rat model (Welsh et al. 2004). For serogroups A, W135 and Y, there is no established threshold of protection.

A first quadrivalent (A, C, W135, Y) meningococcal conjugate vaccine (Menactra ${ }^{\mathrm{TM}}$ ) was licensed in Canada in 2006, and has been approved for use in persons 2 to 55 years of age (Sanofi Pasteur Limited 2006; NACI 2007). One $0.5 \mathrm{~mL}$ dose of this vaccine contains $4 \mu \mathrm{g}$ each of the four polysaccharides conjugated to a total of $48 \mu \mathrm{g}$ of diphtheria toxoid protein carrier. Other products are under development, using either the CRM197 carrier protein derived from the diphtheria toxoid or the tetanus toxoid. The immunologic response to the quadrivalent (A, C, W135, Y) meningococcal diphtheria toxoid conjugate vaccine (Men4-DT) has been investigated in different age groups and different immunologic markers were provided in reports, including the geometric mean SBA (rabbit complement) titre on day 28 and at 6 months, the percentage of subjects who seroconverted (defined as a SBA titre $<1: 8$ on day 0 , and $\geq 1: 32$ on day 28 ), the percentage of subjects with $a \geq 4$-fold rise in SBA titre, and the percentage of subjects with a SBA titre on day $28 \geq 1: 128$ or 1:8 (Sanofi Pasteur Limited 2006; Rennels et al. 2002; Rennels et al. 2004). Generally, the response was better for serogroup A than for other serogroups, and an age-related response was also observed. In infants, the proportion of subjects with SBA titres $\geq 1: 8$ one month after 3 doses was between 92\% for serogroup A and only 54\% for serogroup C (Rennels et al. 2004). In children aged between 12 
and 22 months, two doses were needed for a satisfactory response (Rennels et al. 2002). In subjects aged 2-10 years, the percentage of seroconversion after one dose was $99 \%$ for serogroup A, 96\% for serogroup W135, 88\% for serogroup C, and $86 \%$ for serogroup Y (Pichichero et al., 2005). In another trial, the proportion of seroconverters was $97 \%$ for serogroups A and W135, and 100\% for serogroups $C$ and $Y$ (Lagos et al., 2005). In children aged 2-3 years, Men4-DT induced serogroup $C$ antibodies having higher functional activity than those induced by the quadrivalent polysaccharide vaccine (Granoff et al. 2004). For all serogroups, however, antibody concentrations decreased with time and 2-3 years after Men4-DT administration, many subjects had SBA titres (human complement) below 1:4 (Granoff et al., 2005). However, persistence of immune memory and absence of hyporesponsiveness were demonstrated following administration of one-tenth of a dose of tetravalent polysaccharide vaccine (Pichichero, 2005). In another trial, children aged 2 to 4 years, previously vaccinated more than one year earlier with a monovalent $C$ conjugate vaccine received one dose of Men4-DT and the response to serogroup $C$ was of high magnitude, consistent with a booster response in primed individuals (El Bashir et al., 2006). In adolescents and adults, Men4-DT induced a strong immunologic response with seroconversion rates higher than 90\% for all serogroups (Sanofi Pasteur Limited, 2006). In adolescents, persistence of protective antibodies and of immunologic memory for all serogroups was demonstrated up to 3 years after one dose of Men4-DT (Pichichero, 2005; Vu et al., 2006).

\section{Effectiveness}

All meningococcal conjugate vaccines have been licensed on the basis of immunologic studies and criteria defined by experts (NACI 2007). In all trials in persons aged 2 years or more, short-term immunologic response to Men4-DT was non-inferior to the response to quadrivalent polysaccharide vaccine. Men4-DT generated antibodies having better functional characteristics and longer persistence, while inducing a strong anamnestic response following a booster dose with no hyporesponsiveness (Sanofi Pasteur Limited 2006; NACI 2007). For all these reasons, Men4-DT should be preferred to the quadrivalent polysaccharide vaccine for all indications of a quadrivalent or a serogroup A, Y or W135 meningococcal vaccine in those aged 2 years and older.

The field effectiveness of monovalent serogroup C conjugate vaccines has been evaluated in large post-marketing epidemiological studies, and results indicate a high level of protection in the first year following primary immunization with a variable number of doses in all age groups, sustained protection up to 4 years following administration of one dose in the second year of life or later, but no evidence of sustained protection for those who received a primary immunization series before the age of 6 months (Trotter et al., 2004; De Wals et al., 2004; Larrauri et al., 2005). To date, there is little evidence regarding sustained protection beyond 4 years in those who received monovalent serogroup $\mathrm{C}$ conjugate vaccine in the second year of life or later. Currently, there are no data on the clinical effectiveness of Men4-DT and there has been no immunologic study providing a head-to-head comparison of Men4-DT with field-tested serogroup C conjugate vaccines containing either the CRM197 carrier protein derived from the diphtheria toxoid (Meningitec $^{\mathrm{TM}}$, Wyeth, and Menjugate ${ }^{\mathrm{TM}}$, Novartis Vaccines) or the tetanus toxoid (Neis Vac- $\mathrm{C}^{\mathrm{TM}}$, GlaxoSmithKline). Although the immune response of Menactra ${ }^{\mathrm{TM}}$ was compared to the response of the polysaccharide vaccine, and was non-inferior for serogroup C, it is established that the polysaccharide vaccine provides a low level of protection of short duration for this serogroup in children (De Wals et al., 2005). A case-control study aiming to 
assess effectiveness is underway in the U.S. (CDC, personal communication, 2007). For these reasons, until the results of new studies are available, the three monovalent serogroup $C$ conjugate vaccines currently approved in Canada should be preferred for all indications of vaccination against serogroup C IMD, especially in children.

While the effectiveness of polysaccharide vaccines to prevent serogroup A and C IMD has been demonstrated in experimental and observational studies in adolescents and adults (Frasch 1995; De Wals et al., 2005), immunologic studies have shown that Men4-DT is a more potent vaccine. For this reason, Men4-DT could be considered for routine immunization of adolescents, as recommended in the US (ACIP 2005), including those already immunized with a monovalent serogroup C conjugate vaccine or a polysaccharide vaccine at a young age. The rationale for this booster dose is to ensure that circulating antibodies are present as adolescents enter the peak years of IMD beyond infancy, which are between 15 and 25 years of age. As the incubation period of IMD is short [range 2 to 10 days, commonly 3 to 4 days (Heyman, 2004)] it is now generally accepted that circulating antibodies are necessary to prevent IMD and that the ability to mount a memory response is not sufficient to prevent disease (Auckland et al., 2006; Snape et al., 2006). As well, carriage of meningococci is highest during adolescence (Cartwright, 1995) and preventing carriage in adolescents may have an impact on herd immunity in the community, indirectly protecting infants (Trotter et al., 2005).

\section{Safety}

Vaccines containing proteins are generally associated with slightly more common and severe local reactions than polysaccharide vaccines, and Men4-DT is no exception. However, the experience of administration of Men4-DT in nine clinical trials involving more than 10000 subjects is very reassuring and adverse events were not substantially different than those reported in the control groups vaccinated with a quadrivalent polysaccharide vaccine (Sanofi Pasteur Limited, 2006).

In the US, MenactraTM is widely used for vaccinating adolescents and college students. A cluster of cases of Guillain-Barré syndrome (GBS) was reported in the 6-week period following vaccine administration (Woo et al., 2006). To date, 19 cases of GBS have been documented, mostly in adolescents and young adults. A statistically non-significant risk of approximately one case per million doses has been calculated (NACI, 2007). GBS is an acute autoimmune inflammatory demyelinating neuropathy affecting peripheral motor and/or sensory nerves. The causal mechanisms are still poorly understood (Hahn, 1998; Hughes et al., 2005). In 1976-1977, an excess risk of GBS was identified following the administration of inactivated swine influenza vaccines (Langmuir et al., 1984). However, this association was not seen or was of borderline significance with subsequent influenza vaccine preparations (Hurwitz et al., 1981; Kaplan et al., 1982; Lasky et al. 1998; Roscelli et al., 1991; Haber et al., 2004). In a large study in the UK focusing on GBS following the administration of routine vaccines in all age groups, no association was found (Hughes et al., 2006). In the UK, more than 18 million doses of three serogroup $C$ meningococcal vaccines were administered during the 1999-2001 mass immunization campaign and no cluster of GBS was reported through the passive surveillance system of adverse events (Committee on Safety of Medicines Expert Working Group, 2002). In the province of Quebec, a study was performed to assess the risk of GBS following the administration of a CRM197 meningococcal serogroup $C$ conjugate vaccine in a mass immunization campaign in 2001. 
Immunization records were linked with hospital discharge summaries, and medical records were reviewed. In the cohort of 1.9 million individuals, the frequency of GBS during the post-vaccination period was lower than expected for all comparisons (De Wals P, Deceuninck G, Buucher RM, Ouakki M. Risk of Guillain-Barré syndrome following serogroup $C$ meningococcal conjugate vaccine in Quebec, Canada. Clin Infect Dis, 2008 Apr 15;46(8):e75-7). An association between meningococcal conjugate $C$ vaccines and GBS can be reasonably excluded and a causal association between Men4-DT and GBS has not been established. In the US, a precautionary statement was issued for individuals with a history of GBS, but the recommendation to vaccinate high-risk groups was not altered (Woo et al., 2006).

NACI has reviewed the data on safety of Menactra $^{\mathrm{TM}}$ (CCDR, ...2009). The main concern is about the incidence of Guillan-Barré syndrome (GBS) post immunization. The Centers for Disease Control (CDC) in the United States have also reviewed the safety of Menactra ${ }^{\mathrm{TM}}$. "CDC is unable to determine if Menactra ${ }^{\mathrm{TM}}$ increases a risk of GBS in people who receive the vaccine. GBS is a rare illness, and the expected background population rates of GBS are not known precisely. An ongoing known risk for serious meningococcal disease exists. Therefore, $\mathrm{CDC}$ recommends continuation of current vaccination strategies." (http://www.cdc. gov/vaccinesafety/concerns/gbsfactsheet.htm).

\section{Concomitant Vaccine Administration}

Ideally, new vaccines included in publicly funded programs should be given at the same time as other vaccines to increase coverage levels and minimize administration costs. Concomitant use of Men4-DT with the adult formulation of tetanusdiphtheria (Td) vaccine was assessed and no significant negative interaction was observed (Sanofi Pasteur Limited, 2006). This was the most important association to study because of the presence of the diphteria toxoid antigen in both products. There are currently no data on the concomitant administration of Men4-DT with other vaccines currently offered to adolescents in Canada, including Tdap, hepatitis A and B, and HPV. 


\section{Immunization Strategies}

The goals of immunization programs against IMD should be: i) to prevent the occurrence of outbreaks or epidemics, ii) to control outbreaks or epidemics if not prevented, iii) to prevent sporadic cases and iv) to prevent secondary cases among close contacts of IMD cases.

As presented in Table 1, vaccination against serogroup C meningococcal infections is included in the immunization schedules of all Canadian provinces and territories. For primary immunization, the most frequent recommendation is a single dose of type $C$ conjugate meningococcal vaccine (C-CMV) at 12 months (in effect in New Brunswick, Newfoundland and Labrador, Nova Scotia, Prince Edward Island, Quebec, Ontario,
Saskatchewan and Nunavut). Primary immunization with three early doses (2, 4 \& 6 months) was recommended in Alberta, but this schedule has recently been changed to administer the third dose at 12 months. Two-dose calendars exist in British Columbia ( $2 \& 12$ months), in the Yukon ( $2 \& 6$ months) and in the Northwest Territories ( $2 \& 4$ months). Finally, a calendar offering a single dose at 12 months, with catch up in the 4th year of primary school in Manitoba, a strategy justified by the epidemiological situation in 2004 . Catch-up vaccination with variable schedules exists in most jurisdictions. In Alberta and Quebec, mass immunization campaigns have made any catch-up vaccination unnecessary.

Table 1: Recommendations regarding immunization schedule against invasive serogroup $\mathrm{C}$ meningococcal disease in Canadian provinces and territories (PHAC 2007/12/28)

\begin{tabular}{|c|c|c|}
\hline & Primary Immunization & Catch-up (1 dose)* \\
\hline \multicolumn{3}{|l|}{ Provinces } \\
\hline Alberta & $\begin{array}{l}3 \text { doses: } \\
2,4 \& 12 \text { months }\end{array}$ & $\begin{array}{l}\text { A mass vaccination campaign was conducted in } 2000 \text { - } \\
2002 \text {, targeting the population aged } 2-24 \text { years, and } \\
\text { polysaccharide meningococcal vaccines were used. } \\
\text { Thereafter, conjugate serogroup C vaccines were used } \\
\text { for those younger than } 2 \text { years of age. }\end{array}$ \\
\hline British Columbia & $\begin{array}{l}2 \text { doses: } \\
2 \& 12 \text { months }\end{array}$ & 1 dose in Grade 6 \\
\hline Manitoba & 1 dose at 12 months & 1 dose in Grade 4 \\
\hline New Brunswick & 1 dose at 12 months & Grades $9\left(\right.$ Menactra $\left.^{\mathrm{TM}}\right)$ \\
\hline $\begin{array}{l}\text { Newfoundland \& } \\
\text { Labrador }\end{array}$ & 1 dose at 12 months & Grades 4, 9 \\
\hline Nova-Scotia & 1 dose at 12 months & Grade 7 and between $14 \& 16$ years \\
\hline Ontario & 1 dose at 12 months & Grade 7 and between 15 and 19 years \\
\hline
\end{tabular}




\begin{tabular}{|c|c|c|}
\hline & Primary Immunization & Catch-up (1 dose)* \\
\hline $\begin{array}{l}\text { Prince Edward } \\
\text { Island }\end{array}$ & 1 dose at 12 months & Grade 9 (Menactra $\left.{ }^{\mathrm{TM}}\right)$ \\
\hline Quebec & 1 dose at 12 months & $\begin{array}{l}\text { A mass vaccination campaign was conducted in } \\
2001, \text { targeting the population aged between } 2 \\
\text { months and } 20 \text { years, using mainly type } C \text { conjugate } \\
\text { meningococcal vaccine. }\end{array}$ \\
\hline Saskatchewan & 1 dose at 12 months & Between $4 \& 6$ years, Grade 6 \\
\hline \multicolumn{3}{|l|}{ Territories } \\
\hline $\begin{array}{l}\text { Northwest } \\
\text { Territories }\end{array}$ & 1 dose at 12 months & $\begin{array}{l}\text { A mass vaccination campaign was conducted in } 2004 \text {, } \\
\text { targeting all individuals of school-going age with type } \\
\mathrm{C} \text { conjugate meningococcal vaccine. Catch-up until } 5 \\
\text { years. Screening and catch-up Grade } 9\end{array}$ \\
\hline Nunavut & 1 dose at 12 months & Between 14 and 16 years \\
\hline Yukon & $\begin{array}{l}2 \text { doses: } \\
2 \& 6 \text { months }\end{array}$ & $\begin{array}{l}\text { Grade } 9 \text { catch-up; post secondary students leaving } \\
\text { school and/or not previously vaccinated. }\end{array}$ \\
\hline
\end{tabular}

* A monovalent serogroup C meningococcal conjugate vaccine is used for catch-up, excepted in Prince Edward Island and in New Brunswick where Men4-DT is used.

Men4-DT has not been licensed for use in infants less than 2 years of age, and in this age group the immune response is low, particularly for the C, Y and W135 antigens (Rennels et al., 2002). In Canada, the first increase in meningococcal infection occurs near the age of 1 year and is mainly caused by serogroup C (Figure 1). In addition, there are no provinces or territories that have systematic vaccination at 2 or 3 years of age. For all these reasons, Men4-DT should not be used for primary vaccination against serogroup $C$ in young children. Instead, primary vaccination should be performed with a monovalent conjugate serogroup $C$ vaccine using one dose at the age of 12 months, or eventually at a younger age, using one or two doses with a booster dose in the second year (De Wals et al., 2006).

The preferred indication for Men4-DT in Canada is for vaccination of adolescents at the age of
12 years, just before the increase in incidence of IMD that occurs at the age of 13 years. For adolescents not previously vaccinated, this would be a primary vaccination. For those previously vaccinated with $\mathrm{C}-\mathrm{MCV}$ at a young age, this would be a booster dose for serogroup $\mathrm{C}$ and a primary vaccination for the serogroups $\mathrm{A}, \mathrm{Y}$ and W135. Results of epidemiological studies in the United Kingdom and Spain indicate a progressive decrease in protection during the years following primary vaccination with a serogroup $C$ conjugate vaccine, which is more pronounced if the primary vaccination occurred at a young age (Trotter et al., 2004; Larrauri et al., 2005). Modeling of the relative effectiveness of various vaccination schedules suggests that revaccination near the age of 12 years is useful when the decrease of protection from primary vaccination at a young age is equal to or greater than $3 \%$ per year (De Wals 
et al., 2006). For protection against serogroup C, addition of revaccination around 10 years after a primary vaccination could offer additional duration of protection. In fact, observed concentrations of bactericidal antibodies against serogroup $C$ are 35 times higher one month after revaccination with Men4-DT than after primary vaccination (Pichichero et al., 2005; El Bashir et al., 2006).

Like the conjugate serogroup C vaccine, Men4-DT probably induces appearance of mucosal antibodies (Zhang et al., 2000; Zhang et al., 2002). Therefore, vaccination of an important proportion of adolescents could significantly reduce transmission of pathogenic strains in the entire population, as the prevalence of carriage of $N$. meningitidis is maximal in this age group (Cartwright, 1995). In the United Kingdom, following a mass immunization campaign using C-MCV (reaching 81\% of the population aged 18 years and under), observed carriage of serogroup $\mathrm{C}$ was reduced from $4.5 \%$ to $1.5 \%$ in students 15 to 17 years of age (Maiden et al., 2002), while the incidence of serogroup C IMD decreased by 52\% in the non-vaccinated fraction of the target population (Ramsay et al., 2003). No epidemiological data exist regarding herd immunity induced by a routine vaccination program when only one or two cohorts are vaccinated per year. Trotter and coworkers (2005) developed a dynamic simulation model of meningococcal infection and disease that predicts a maximal indirect effect when adolescents are immunized with conjugate meningococcal vaccine. This herd immunity effect could be quite significant following a revaccination against serogroup $C$ and could prevent new outbreaks. Regarding the other serogroups, herd immunity would be induced by vaccination of adolescents with Men4-DT, but a group of susceptible individuals would remain in children and older adults.

In the event of licensure of a quadrivalent meningococcal conjugate vaccine for children under 2 years of age which demonstrates a non-inferior immune response to the serogroup $\mathrm{C}$ polysaccharide as compared with currently available monovalent $C$ vaccines, the ideal strategy would shift to the exclusive use of the quadrivalent product both for the priming (using either one dose at 12 months, or one or two doses below one year followed by a booster dose in the second year) and for the booster adolescent dose (ideally at 12 years). 


\section{Cost Effectiveness}

IMD cases are not frequent in Canada but are associated with substantive societal costs and losses of healthy life years (De Wals et al., 2007). Average short-term medical costs per IMD case have been estimated at $\$ 13,000$ and the annual treatment costs for a survivor with permanent physical sequelae at $\$ 17,000$ below 18 years of age and $\$ 4,000$ at 18 years of age and over (De Wals et al., 2007). In addition, indirect costs related to productivity losses caused by premature death or permanent physical sequelae should be taken into account.

New vaccines have somewhat of a disadvantage compared with older products, whose development costs have already been paid off. In particular, multivalent conjugate vaccines are more complex to produce than monovalent products. Also, the production capacity of Men4-DT is limited while there are three production facilities for monovalent serogroup $C$ products. For publicly funded immunization programs, provinces and territories invite vaccine manufacturers to submit proposals (usually for short-term contracts), and the company offering the lowest price is selected. This purchasing process results in very low prices in a market where there is fierce competition, as was observed with MenC-C, the cost of which rapidly decreased from $\$ 50$ to under $\$ 20$. The expected market price of Men4-DT for the public sector in Canada is $\$ 70$ per dose (De Wals et al., 2007). In the future, however, the differential price between monovalent $C$ and quadrivalent conjugate vaccines may decrease, especially when several quadrivalent products enter the market.

Costs associated with these potential vaccination programs mainly include vaccine purchase and administration costs. In a recent cost-effectiveness analysis, the marginal opportunity costs for the administration of Men4-DT vaccine to a teenager along with another recommended vaccine were estimated to $\$ 8.50$ (De Wals et al., 2007). Using this value and a vaccine purchase price of $\$ 70$ per dose, program costs for provincial governments according to different levels of vaccine coverage of teenagers are shown in Table 2. Overall, program costs would range between $\$ 19$ and $\$ 29$ million. Vaccine delivery and administration costs in Canadian territories may be higher than in more densely populated provinces and there are no good data to provide reliable estimates of total program costs in these regions. Adverse reactions associated with the administration of meningococcal conjugate vaccines in adolescents requiring medical attention are rare, and associated costs do not affect total program costs significantly (De Wals et al., 2007).

Table 2: Estimated program cost* to the health system according to different population coverage of teenagers with a quadrivalent meningococcal conjugate vaccine in Canada

\begin{tabular}{|l|c|c|c|c|c|}
\hline \multirow{2}{*}{\multicolumn{1}{|c|}{ Province }} & \multirow{2}{*}{$\begin{array}{c}\text { Population } \\
\text { (2006 estimate) }\end{array}$} & \multicolumn{4}{c|}{ Vaccination Coverage } \\
\cline { 5 - 6 } & & $\mathbf{6 0 \%}$ & $\mathbf{7 0 \%}$ & $\mathbf{8 0 \%}$ & $\mathbf{9 0 \%}$ \\
\hline Newfoundland and Labrador & 6000 & $\$ 283$ & $\$ 330$ & $\$ 377$ & $\$ 424$ \\
\hline Prince Edward Island & 2000 & $\$ 94$ & $\$ 110$ & $\$ 126$ & $\$ 141$ \\
\hline Nova Scotia & 11000 & $\$ 518$ & $\$ 604$ & $\$ 691$ & $\$ 777$ \\
\hline
\end{tabular}




\begin{tabular}{|l|c|r|r|r|r|}
\hline \multirow{2}{*}{ Province } & \multirow{2}{*}{$\begin{array}{c}\text { Population } \\
\text { (2006 estimate) }\end{array}$} & \multicolumn{4}{|c|}{ Vaccination Coverage } \\
\cline { 3 - 6 } & & $\mathbf{6 0 \%}$ & $\mathbf{7 0 \%}$ & $\mathbf{8 0 \%}$ & \multicolumn{1}{c|}{$\mathbf{9 0 \%}$} \\
\hline New Brunswick & 9000 & $\$ 424$ & $\$ 495$ & $\$ 565$ & $\$ 636$ \\
\hline Quebec & 95000 & $\$ 4475$ & $\$ 5220$ & $\$ 5966$ & $\$ 6712$ \\
\hline Ontario & 162000 & $\$ 7630$ & $\$ 8902$ & $\$ 10174$ & $\$ 11445$ \\
\hline Manitoba & 16000 & $\$ 754$ & $\$ 879$ & $\$ 1005$ & $\$ 1130$ \\
\hline Saskatchewan & 14000 & $\$ 659$ & $\$ 769$ & $\$ 879$ & $\$ 989$ \\
\hline Alberta & 45000 & $\$ 2120$ & $\$ 2473$ & $\$ 2826$ & $\$ 3179$ \\
\hline British Columbia & 51000 & $\$ 2402$ & $\$ 2802$ & $\$ 3203$ & $\$ 3603$ \\
\hline All 10 provinces & 411000 & $\$ 19358$ & $\$ 22584$ & $\$ 25811$ & $\$ 29037$ \\
\hline
\end{tabular}

$*$ Costs are expressed in thousands of Canadian \$

Currently, the most attractive option for use of Men4-DT in Canada would be vaccination around 12 years of age of naïve subjects or revaccination of subjects primed with a monovalent serogroup $\mathrm{C}$ conjugate vaccine (MenC-C) at a young age. A simulation model was developed for assessing both the direct and indirect effects of a booster dose at 12 years of age with either a monovalent $C$ or a quadrivalent ACYW135 meningococcal conjugate vaccine in a cohort of Canadians immunized at 12 months with Men C-C (De Wals et al., 2007). Age and serogroup-specific incidence and fatality rates were derived from Canadian surveillance data. Vaccine efficacy was estimated from data from the UK and Spain, assuming an age-dependent decline of vaccine efficacy over time. Expected vaccine coverage rates were $90 \%$ at 12 months, and $70 \%$ at 12 years. Herd immunity was modeled using UK data. Vaccine purchase price per dose was $\$ 23$ for MenC-C and $\$ 70$ for Men4DT. Costs and health outcomes were discounted at 3\% per year. Results, expressed in 2004 Canadian dollars and from a societal perspective, were presented for a steady-state situation and a population of one million.

As seen in Table 3, 5.7 cases of vaccine-preventable meningococcal disease would occur each year under the "no vaccination" base scenario. Vaccination at 12 months using MenC-C would reduce the burden of disease by $32 \%$. Adding MenC-C at 12 years of age would reduce the number of cases by $55 \%$ at no marginal cost (a minor saving is predicted). Using Men4-DT for the booster dose would result in a disease reduction of $78 \%$ for a marginal cost of $\$ 31000$ per QALY gained compared to one dose of MenC-C at 12 months. Comparing Men4-DT with MenC-C as a booster dose, the incremental costeffectiveness ratio would be $\$ 113000$ per QALY. 
Table 3: Cost-effectiveness of different immunization strategies using the serogroup $\mathrm{C}$ meningococcal conjugate vaccine (MenC-C) or quadrivalent meningococcal conjugate vaccine (Men4-DT) in a population of one million in Canada, in base model scenario (De Wals et al., 2007).

\begin{tabular}{|c|c|c|c|c|}
\hline Outcome & $\begin{array}{c}\text { No } \\
\text { vaccination }\end{array}$ & $\begin{array}{l}\text { MenC-C at } \\
12 \mathrm{mo}\end{array}$ & $\begin{array}{c}\text { MenC-C at } 12 \mathrm{mo} \\
\quad \& 12 \mathrm{yr}\end{array}$ & $\begin{array}{c}\text { MenC-C at } 12 \mathrm{mo} \& \\
\text { Men4-DT at } 12 \mathrm{yr}\end{array}$ \\
\hline Number doses MenC-C & - & 12000 & 21000 & 12000 \\
\hline Number doses MenC-4 & - & - & - & 9000 \\
\hline Program cost & - & $\$ 316000$ & $\$ 604000$ & $\$ 1033000$ \\
\hline IMD cases averted (direct) & - & 1.0 & 1.7 & 2.0 \\
\hline IMD cases averted (indirect) & - & 0.6 & 2.2 & 2.8 \\
\hline Residual IMD cases & 5.7 & 4.1 & 1.8 & 0.9 \\
\hline \multirow[t]{2}{*}{$\begin{array}{l}\text { Incremental net cost/QALY } \\
\text { ( } 95 \% \text { confidence interval) }\end{array}$} & - & Ref & $\begin{array}{r}\$-1000 \\
(\$-15000 \text { to } \\
\$ 82000)\end{array}$ & $\begin{array}{r}\$ 31000 \\
(\$ 12000 \text { to } \\
\$ 111000)\end{array}$ \\
\hline & & & Ref & $\begin{array}{r}\$ 113000 \\
(\$ 72000 \text { to } \\
\$ 198000)\end{array}$ \\
\hline
\end{tabular}

Results of sensitivity analyses showed that the vaccine effectiveness and the differential price between the two vaccines were the parameters having the strongest impact on the cost/QALY ratios. Any increase in the incidence of serogroup Y would also improve the marginal cost-effectiveness ratio associated with Men4-DT. In all scenarios, however, revaccination with MenC-C was associated with more favourable cost-effectiveness indices than with Men4-DT, and in the current epidemiological situation the differential price between the two vaccines would have to be markedly reduced to reverse this conclusion.

Different simulation models of variable complexity have been developed to analyse the future impact of different immunization strategies (Trotter at al., 2002; De Wals et al. 2004; Trotter et al., 2005; De Wals et al., 2006; De Wals et al., 2007; Caro et al. 2007). There are significant limitations in these models, the most important being the unpredictable epidemiology of Neisseria meningitidis, uncertainty regarding the long term effectiveness of meningococcal conjugate vaccine, and the level of herd immunity provided by routine vaccination. Results can thus provide a rough comparison of the relative impact of different strategies within a specified model but comparisons between models and absolute costeffectiveness indices should be regarded with great care. 


\section{Program Acceptibility}

The theory of risk perception of Slovic (1987) predicts that the level of anxiety and the probability to take action to reduce a risk are more elevated if the source is not well known to those exposed, nor to experts; if the exposure is involuntary and difficult to control; and finally if the consequences are devastating. Invasive meningococcal disease possesses all of these characteristics and it is therefore not surprising to find this disease among the priorities for prevention in the population. A survey of parents of children under 7 years of age in Canada revealed that meningitis was the most feared vaccine-preventable pathology (Ipsos Reid, personal communication, 2001). In the same study, the possibility of prevention, recommendations by health care professionals, and the safety and effectiveness of the vaccine were cited as the most important factors in the decision to vaccinate one's child. Occurrence of an outbreak increases anxiety and the need for intervention, as was observed in Quebec in 1990-1993 and also in 2001 (De Wals et al., 2003). A survey conducted in the region of Sherbrooke, Quebec in 2002 revealed that the large majority of those questioned approved of the mass immunization campaign which had just been completed and nearly $80 \%$ agreed with the inclusion of meningococcal vaccine in the regular vaccination schedule (De Wals et al., 2002). It is therefore logical to predict that the inclusion of a safe and effective vaccine against invasive meningococcal disease in the regular immunization schedule of adolescents in Canada would be well received by the majority of the population and health care professionals. Experience has shown that anti-vaccination groups are less active when there is a social consensus and an absence of controversy regarding a particular vaccine.

It is important to be proactive and circulate objective information regarding the occurrence of a cluster of cases of Guillain-Barré syndrome following administration of Menactra ${ }^{\mathrm{TM}}$. An analogy can be found with the inactivated influenza vaccine. In 2004, an Institute of Medicine review concluded that the evidence concerning a relationship between influenza vaccines other than the swine influenza vaccine was inconclusive (Stratton et al., 2004). Information on this potential risk of very small (if any) magnitude is included in the monographs given to vaccine recipients (MSSS, 2004). However, a survey in the Sherbrooke region in 2000 showed that none of the respondents, $59 \%$ of whom had been vaccinated, cited GBS as one of the possible side effects of the influenza vaccine, suggesting that the information was not transmitted orally (De Wals, 2000). During the interviews, it was relatively easy to explain the syndrome and the mortality risk involved (approximately 10\%). On the other hand, it was much more difficult to explain the significance of the 10,000 to 1 ratio which exists between the annual mortality risk attached to influenza if one is not vaccinated (approximately 1/1,000) and the risk of dying of GBS following vaccination against influenza (approximately 1/10,000,000). As for determining if providing information regarding the risk of GBS could have an effect on their willingness to be vaccinated the following year, $89 \%$ said no, $7 \%$ said yes, and $4 \%$ were uncertain. Among those who had not been vaccinated, the very fact of being informed of the existence of such a risk would reinforce their decision to not be vaccinated in $35 \%$ of the cases. Persons aged 65 years or more may be willing to accept some risk associated with an effective vaccine but the reaction of adolescents and their parents may be completely different. While informing recipients of Menactra ${ }^{\mathrm{TM}}$, it would be important to balance the duty of informed consent with the danger of generating unfounded concerns and the avoidance of an effective preventive intervention. Multiple 
injections can be a challenge for all age groups. To minimize these potential impacts, it is important to plan the adolescent vaccination schedule to avoid the need to administer more than 3 injections per visit. The recent introduction of many new vaccines in Canada has increased the workload for vaccinators significantly. To avoid dissatisfaction of personnel following the announcement of a new program, it is essential to prepare the terrain well in advance and supply adequate resources to persons and organizations involved so they can accomplish this task. If all of these conditions are present, it can be expected that coverage rates in the target population will be high, as is the case in the majority of public immunization programs in Canada (Health Canada, 1997). 


\section{Program Feasibility}

Ideally, vaccination against IMD targeting adolescents should occur between 11 and 13 years of age, before lifestyle changes cause an increase in asymptomatic carriage rates of N. meningitidis, which peak between 15 and 20 years (Cartwright, 1995). The risk of invasive disease also increases from the age of 13 years, reaching a maximum at the age of 18 years (Figure 1). Administration of Men4-DT at the age of 9 or 10 years would risk having suboptimal protection during the high-risk period that lasts until 24 years of age. In contrast, vaccination in the Grade 9 (near 14 years of age), while being slightly too late is still an acceptable option. The expected coverage of the target age group is another factor that should be taken into consideration to select the best strategy.

All Canadian provinces and territories have vaccination programmes targeting young people in these categories (Table 4). Until present, the priority has been immunization of young infants. The multiplication of vaccines available for adolescents requires that more attention be paid to the optimal schedule for this age group. The number and timing of booster doses for diphtheria, tetanus, and pertussis must be re-examined. A school-based program targeting students in the first year of secondary school at the age of 12 years would certainly have advantages regarding the prevalence of naïve subjects, immune response, presence at school, obtaining parental consent, and acceptability of vaccination compared to a program targeting those 14 years of age or older. A quasiexperimental study in Quebec found that a schoolbased vaccination program was the most effective and least costly method to reach this age group (Guay et al., 2003). It can be expected that bringing a 10-14 year-old to a doctor's office or health centre for vaccination may be challenging for parents.

It is necessary to optimize vaccinations in this age group, taking into account the specific constraints and existing programs in each jurisdiction.

Immunization for meningococcal disease (1 dose), diphtheria tetanus and pertussis (1 dose), hepatitis A and B (possibly 2 doses) and HPV (1 to 3 doses) could be completed in 2 or 3 sessions concentrated during the same year.

Table 4: Vaccination programs for 9 to 16 year-olds in Canadian provinces and territories (PHAC 2007b)

\begin{tabular}{|l|l|l|}
\hline \multicolumn{2}{|c|}{ Hepatitis B } & \multicolumn{1}{c|}{ TdaP } \\
\hline Provinces & Grade 5 (3 doses) & Grade 9 \\
\hline Alberta & & Grade 9 \\
\hline British Columbia & Grade 4 (3 doses) & Grade 8 or 9 \\
\hline Manitoba & & Grade 9 \\
\hline New Brunswick & Grade 4 (3 doses) & Grade 9 \\
\hline Newfoundland \& Labrador & Grade 4 (3 doses) & Grade 9 \\
\hline Nova-Scotia & Grade 7 (2 doses) & 14 -16 years \\
\hline Ontario & & \\
\hline
\end{tabular}




\begin{tabular}{|l|l|l|}
\hline & Hepatitis B & \multicolumn{1}{c|}{ TdaP } \\
\hline Prince Edward Island & & Grade 9 \\
\hline Quebec & Grade 4 (3 doses) & Grade 9 (3 ${ }^{\text {rd }}$ year high school) \\
\hline Saskatchewan & Grade 6 (3 doses) & Grade 8 \\
\hline Territories & \multicolumn{2}{|l}{} \\
\hline Northwest Territories & Grade 9 \\
\hline Nunavut & Grade 9 \\
\hline Yukon & & Grade 9 \\
\hline
\end{tabular}

Finally, problems in vaccine supply can occur, especially when only one vaccine is licensed and when there is only one site of production and production line. In the United States, a shortage occurred with Menactra ${ }^{\mathrm{TM}}$ at the beginning of the year 2006, which led to rationing of available vaccine (CDC 2006). The manufacturer announced that the situation would be corrected quickly however this type of incident can be expected to occur as long as only one quadrivalent conjugate meningococcal vaccine is licensed. 


\section{Program Evaluation}

Evaluation of newly implemented immunization programs is the duty of public health authorities and appropriate resources should be invested in monitoring systems and ad hoc studies. For Men4DT, most important issues include the quality of immunization services, vaccination coverage of the target population and global impact of the program on the epidemiology of IMD. In every jurisdiction, surveys should be performed at regular intervals to evaluate quality of information provided to consumers, respect of their freedom of choice and their degree of satisfaction with the service they received. Vaccination coverage of the target population should be assessed through high quality immunization registries or information systems allowing identification of administration of specific vaccines to specific persons. Postal or telephone surveys are alternative methods to assess vaccination coverage, while allowing identification of barriers and factors favouring vaccine uptake. Finally, impact of the immunization program on epidemiology of IMD should be monitored prospectively. For this, linkage of notification data with laboratory data and hospital discharge summary records is important to ensure a high level of ascertainment of IMD cases. Ideally, immunization status of every incident IMD case should be assessed in order to identify vaccine failures and their potential causes. Systematic genotypic and phenotypic characterization of strains of $N$. meningitidis by provincial and national reference laboratories is also required. Capsular switching and clonal replacement have been described following the implementation of new vaccines (Alonso et al., 2007) and this is a threat to be considered with Men4-DT. 


\section{Research Questions}

Until now, there has been no trial comparing the immunologic response to the serogroup $C$ component of the Men4-DT with monovalent serogroup $C$ conjugate vaccines using other protein carriers. In a head-to-head comparison of one dose of 3 different monovalent serogroup $\mathrm{C}$ meningococcal conjugate vaccines in toddlers in the UK, a significantly higher antibody response was observed with a de-O-acetylated $\mathrm{C}$ polysaccharide-tetanus toxoid formulation than with O-acetylated C oligosaccharide-CRM197 formulations (Richmond et al., 2001). For this reason, a two-dose schedule for the product using the tetanus toxoid carrier protein has been approved for the routine immunization of infants, instead of 3 doses for the products using the CRM197 protein carrier (NACI, 2005). It will be important to compare the relative immunogenicity of different meningococcal vaccines and different vaccination schedules, taking advantage of the existing variability of routine immunization schedules in Canada. The non-inferiority of the immune response of quadrivalent vaccines as compared with currently available monovalent $C$ conjugate vaccines should be demonstrated, especially for children less than 11 years of age. Also, the marginal benefit of one, two or three doses for primary immunization should be assessed in studies including a follow-up of several years (ideally up to 12 years of age).

Evaluation of protection conferred by meningococcal vaccines is challenging in an endemic situation and the method of choice is the retrospective case-control study. This allows the control of confounding factors which is very important when high immunization rates are achieved, implying significant differences in the characteristics of vaccinated and non-vaccinated individuals (Orenstein et al., 1988; De Wals et al., 2005). Statistical power is the most problematic issue, and the possibility of a multiprovincial study and collaboration with a similar initiative in the US should be considered.

Studies are currently underway to determine the need for booster immunization with Menactra ${ }^{\mathrm{TM}}$. Results are expected in 2010. To evaluate this program better in 2010, further work in the following areas is needed:

1. Monitoring and surveillance for the disease;

2. Economic analysis to assess the comparative benefit of Menactra ${ }^{\mathrm{TM}}$ versus other meningococcal vaccines and also in relation to various schedules;

3. Immunogenicity and duration of antibody response of different meningococcal vaccines and different vaccination schedules. For example:

- $\quad$ Meningococcal C conjugate (MCC) at 12 months of age (P/T cohorts immunized since 2001)

- MCC at 12 - 14 years of age (P/Ts with school catch-up programs)

- MCC at 12 months and boosted with MCC at $12-14$ years of age

- Quadrivalent meningococcal conjugate vaccine booster at 12 - 14 years of age to those who received MCC at 12 months

- Quadrivalent meningococcal conjugate vaccine to meningococcal vaccine naive 12 - 14 year olds 
4. Comparison of immune response of quadrivalent conjugate vaccines as compared with MCC vaccines in children less than 11 years of age.
5. Evaluation of meningococcal vaccine effectiveness, vaccine impact on nasopharyngeal carriage of meningococci, and indirect effect of vaccine on disease rates among unvaccinated populations. 


\section{Other Considerations}

\section{Equity}

The current situation in Canada with marked differences in recommended schedules for routine and catch-up vaccination against serogroup C can be regarded as inequitable. Introduction of a systematic vaccination or revaccination of adolescents will maximize protection, reduce inequities, and harmonize practices. From a program perspective, the announcement of a new vaccination program targeting adolescents and providing individual protection against a deadly disease, while minimizing the probability of new outbreaks or epidemics at the population level, without posing any unacceptable risk would certainly be well received by the population.

\section{Ethical Consideration}

Menactra ${ }^{\mathrm{TM}}$ is a safe and effective vaccine. However, as with other vaccines and medication, it is not perfectly safe nor perfectly effective.

Decisions about use of vaccines are based on the relative balance of risks and benefits. Decision for the introduction of this vaccine should be based on careful consideration of the risk of serogroups

A, Y, and W-135 in a province or territory and the cost benefit analysis for prevention of disease.

\section{CIC Recommendation for Use of Meningococcal Vaccines}

The National Advisory Committee on Immunization (NACI) published detailed recommendations for the use of meningococcal vaccines currently authorized for use in Canada (CIG pp237-250 and CCDR, vol. 33, and Nov. 2007). An update was also published recently in the CCDR, vol. These recommendations are updated as new information becomes available.

Based on the analysis provided in this paper by Dr. De Wals (September 2007 report to PHAC) and the scientific evaluation by NACI, CIC concurs with the recommendations provided by NACI for the use of the meningococcal vaccines in the following manner.

Goal: To reduce illness and death due to Neisseria meningitidis ( $N$ meningitidis) sero-group $\mathrm{C}$ through immunization (Consensus conf. 2005). 


\section{Recommended Immunization Program}

Further to information provided in this paper and scientific and epidemiological information provided by NACI, CIC in concurrence with NACI, provides the following advice for the use of meningococcal vaccines in publicly funded programs.

\begin{tabular}{|l|l|l|}
\hline \multicolumn{1}{|c|}{ Age Group } & \multicolumn{1}{|c|}{$\begin{array}{c}\text { Preferred Vaccine for } \\
\text { Consideration }\end{array}$} & \multicolumn{1}{|c|}{ Booster } \\
\hline Early Childhood & $\begin{array}{l}\text { Meningococcal Conjugate C } \\
\text { (Men C-C) }\end{array}$ & $\begin{array}{l}\text { An early adolescent booster dose } \\
\text { is recommended with either Men } \\
\text { C-C or Men4-DT. }\end{array}$ \\
\hline $\begin{array}{l}\text { Pre-Adolescent } \geq 5 \text { years of age } \\
\text { and up to 12 years }\end{array}$ & $\begin{array}{l}\text { Meningococcal Conjugate C } \\
\text { (Men C-C) if not received in } \\
\text { early childhood }\end{array}$ & $\begin{array}{l}\text { Decision will have to be made } \\
\text { when more data is available for } \\
\text { duration of protection. }\end{array}$ \\
\hline Adolescent (Age 12 and higher) & Men C-C.or Men4-DT & More data is needed \\
\hline
\end{tabular}

A routine early childhood immunization program with MenactraTM is not advised for consideration at this time. An early booster adolescent dose with Men C-C or Men4-DT should be considered. In the provinces/territories that currently offer adolescent catch-up programs, the most practical approach would be to turn the catch-up into booster doses when previously vaccinated early childhood cohorts reach adolescent The choice of the products (Men C-C or Men4-DT) will be made by $\mathrm{P} / \mathrm{T}$ based on the epidemiology of $\mathrm{A}, \mathrm{Y}$ and W-135. The cost-effectiveness of the strategy on implementing a program at adolescence is not available at this stage. However, provinces/ territories that don't have any catch-up programs might wish to use a very simple measure to help them appreciate the cost of implementing an adolescent program. Using their epidemiological data and assuming life-long immunity, the cost per number of IMD cases prevented, by year and by sérogroupe can be calculated, using either the Men C-C or the Men4-DT vaccine. 


\section{Conclusion}

It appears that vaccination of young adolescents with Men4-DT could contribute substantially to the prevention of serogroup A, C, Y \& W135 invasive meningococcal disease in the Canadian population, for sporadic cases as well as for outbreaks (which have generally been caused by virulent strains of serogroup $C$ during the last two decades). Such a program would complete primary vaccination at a young age with monovalent conjugate serogroup $C$ vaccine, which is already in place in most provinces and territories. In the current situation, revaccination of young adolescents with Men4-DT would offer advantages in reduction of mortality and morbidity for a reasonable cost, estimated at $31000 \$$ per QALY gained compared to 1 dose of MenC-C at 12 months. In the event of an outbreak, Menactra ${ }^{\mathrm{TM}}$ is preferred.

In the event of licensure of a quadrivalent conjugate vaccine for use in infants, a schedule with two doses, the first at 12 months and the second at 12 years of age, would probably be the most cost-effective strategy. A more expensive but only slightly more effective strategy would be a program with 3 doses at 2 months, 12 months, and 12 years. A schedule with 2 doses at a young age followed by a booster dose during the second year and another during adolescence would probably be the most effective but also by far the most costly strategy. Immunogenicity studies comparing different schedules of new quadrivalent meningococcal conjugate vaccines should be performed.

Ideally, meningococcal vaccination during adolescence should be provided at 12 years of age, throughout a school-based program (Grade 7) and combined with other vaccinations (Tdap and HPV for example). Revision of all vaccination programs for adolescents in Canada is needed, including consideration of immunization schedules as well as modes of vaccine administration. Vaccination of adolescents against meningococcal disease should be well received by the population and health professionals who deal with fulminant invasive meningococcal infections with dramatic consequences. From a program perspective, this decision should be well received, not extremely expensive relative to other health programs, and free of any major risks considering current knowledge. Ultimately, the decision to use Men4-DT or a monovalent serogroup $C$ product is an issue of provincial/territorial priorities based on the local epidemiology of $\mathrm{A}, \mathrm{Y}$, and $\mathrm{W} 135$. 


\section{References}

ACIP (2005). Prevention and control of meningococcal disease. Recommendations of the Advisory Committee on Immunization Practices. MMWR 54 ( RR-7) : 1-21.

Alonso JM et al. (2007). Workshop on vaccine pressure and Neisseria meningitidis, Annecy, France, 9-11 March 2005. Vaccine 25 : 4125-9.

Andrews N, Borrow R, Miller E (2003). Validation of serological correlate of protection for meningococcal $\mathrm{C}$ conjugate vaccine by using efficacy estimates from postlicensure surveillance in England. Clin Diagn Lab Immunol 10 : 780-786.

Ashton FE, Caugant DA (2001). The panmictic nature of Neisseria meningitidis serogroup B during a period of endemic disease in Canada. Can J Microbiol 47 : 283-89.

Auckland C, Gray S, Borrow R et al. (2006). Clinical and immunologic risk factors for meningococcal $C$ conjugate vaccine failure in the United Kingdom. JID 194: 1745 - 52.

Borrow R, Balmer P, Miller E (2005).

Meningococcal surrogates of protection-serum bactericidal antibody activity. Vaccine 23 : 2222-7.

Caro JJ, Möller J, Getsios D et al. (2007). Invasive meningococcal disease epidemiology and control measures: a framework for evaluation. BMC Public Health $7: 130$.

Cartwright K (1995). Meningococcal carriage and disease. In. Meningococcal disease. Ed. Cartwright K. Chichester, U.K. : John Wiley \& Sons : 71-114.

CDC (2006). Notice to readers : Improved supply of meningococcal conjugate vaccine, recommendation to resume vaccination of children aged 11-12 years. MMWR 55 (43) : 1177.

Committee on Safety of Medicines Expert Working Group. (2002). Report of the Committee on Safety of Medicines Expert Working Group on Meningococcal Group C Conjugate Vaccines. London, UK, Department of Health.

De Wals P (2000). Does informing patients of the risk of acquiring Guillain-Barré syndrome following influenza vaccination have an effect on their willingness to be vaccinated ? CCDR 26: 205-7.

De Wals P (2006). Immunization strategies for the control of serogroup $\mathrm{C}$ meningococcal disease in developed countries. Expert Rev Vaccines 5 : 269-75.

De Wals P, Allard MA, Guindon K et al. (2002). Is vaccination against meningitis useful ? The findings of an investigation in the Sherbrooke region. CCDR $28: 61-63$.

De Wals P, Coudeville L, Trottier P et al. (2007). Vaccinating adolescents against meningococcal disease in Canada: a cost-effectiveness analysis. Vaccine 25 : 5433-40.

De Wals P, Deceuninck G, Boulianne N et al. (2004). Effectiveness of a mass immunization campaign using serogroup $C$ meningococcal conjugate vaccine. JAMA 292 : 2491-2494.

De Wals P, Deceuninck G, De Serres G et al. (2005). Effectiveness of serogroup $C$ meningococcal polysaccharide vaccine: results from a case-control study in Quebec. Clin Infect Dis 40 1116-22.

De Wals P, Duval B, De Serres G et al. (2003). Santé publique : le contrôle des maladies 
méningococciques au Québec. Médecine/Sciences 19 : 1011-5.

De Wals P, Nguyen VH, Erickson LJ et al. (2004). Cost-effectiveness of immunization strategies for the control of serogroup $\mathrm{C}$ meningococcal disease. Vaccine 22 : 1233-40.

De Wals P, Trottier P, Pépin J (2006). Relative efficacy of different immunization schedules for the prevention of serogroup $C$ meningococcal disease: a model-based evaluation. Vaccine 24 : 3500-4.

Deeks S, Kertesz D, Ryan A et al. (1997).

Surveillance of invasive meningococcal disease in Canada, 1995-1996. Can Commun Dis Rep 23-16: 121-125.

El Bashir H, Heath PT, Papa T et al. (2006). Antibody responses to meningococcal (groups A, C, Y and W135) polysaccharide diphtheria toxoid conjugate vaccine in children who previously received meningococcal $C$ conjugate vaccine. Vaccine 24: 2544-2549.

Erickson L, De Wals P (1998). Complications and sequelae of meningococcal disease in Quebec, Canada, 1990-1994. Clin Infect Dis 26:1159-64.

Erickson LJ, De Wals P, Farand L (2005). An analytical framework for immunization program planning in Canada. Vaccine 23 : 2468-74.

Frasch CE (1995). Meningococcal vaccines: past, present and future. In: Cartwright K, ed. Meningococcal disease. Chichester: John Wiley and Sons : 245-83.

Girard MP, Preziozi MP, Aguado MT et al. (2006). A review of vaccine research and development: Meningococcal disease. Vaccine 24 : 4692-4700.
Goldschneider I, Gotschlich EC, Artenstein MS (1969). Human immunity to the meningococcus. I. The role of humoral antibodies. J Exp Med 129 : $1307-26$.

Granoff DM, Harris SL (2004). Protective activity of group $C$ anticapsular antibodies elicited in two-year-olds by an investigational quadrivalent Neisseria meningitidis-diphtheria toxoid conjugate vaccine. Pediatr Infect Dis J 23 : 490-7.

Granoff DM, Morgan A, Welsch JA (2005). Immunogenicity of an investigational quadrivalent Neisseria meningitidis-diphtheria toxoid conjugate vaccine in 2-year old children. Vaccine 23 : 43074314.

Granoff DM, Morgan A, Welsch JA (2005).

Persistence of group $C$ anticapsular antibodies two to three years after immunization with an investigational quadrivalent Neisseria meningitidisdiphtheria toxoid conjugate vaccine. Pediatr Infect Dis J 24 : 132-36.

Guay M, Clouâtre AM, Blackburn M et al. (2003). Effectiveness and cost comparison of two strategies for hepatitis B vaccination of schoolchildren. Can J Public Health 94 : 64-7.

Haber P, DeStefano F, Angulo FJ et al (2004). Guillain-Barré syndrome following influenza vaccination. JAMA 292 : 2478-81.

Hahn A (1998). Guillain-Barré syndrome. Lancet $352: 635-41$.

Harrison LH, Jolley KA, Shutt KA et al. (2006). Antigenic shift and increased incidence of meningococcal disease. J Infect Dis 193 : 1266-74.

Health Canada (1997). Canadian report on immunization, 1996. CCDR 23 S4. Available 
at : http://www.phac-aspc.gc.ca/publicat/ccdrrmtc/97vol23/23s4/index.html

Hughes RA, Charlton J, Latinovic R et al. (2006). No association between immunization and Guillain-Barré syndrome in the United Kingdom, 1992 to 2000. Arch Intern Med 166 : 1301-4.

Hughes RA, Cornblath DR (2005). Guillain-Barré syndrome. Lancet $366:$ 1653-66.

Hurwitz ES, Schonberger LB, Nelson DB et al. (1981). Guillain-Barré syndrome and the 19781979 influenza vaccine. N Engl J Med 304 : 1557-61.

Kaplan JE, Katona P, Hurwitz ES et al. (1982). Guillain-Barré syndrome in the United States, 1979-1980 and 1980-1981. Lack of an association with influenza vaccination. JAMA 248 : 698-700.

Lagos R, Papa T, Mu oz A et al. (2005). Safety and immunogenicity of a meningococcal (groups A, C, Y, W-135) polysaccharide diphtheria toxoid conjugate vaccine in healthy children aged 2 to 10 years in Chile. Human Vaccines $1: 228-31$.

Langmuir AD, Bregman DJ, Kurland LT et al. (1984). An epidemiologic and clinical evaluation of Guillain-Barré syndrome reported in association with the administration of swine influenza vaccines. Am J Epidemiol 119 : 841-79.

Larrauri A, Cano R, Garcia M et al. (2005). Impact and effectiveness of meningococcal $\mathrm{C}$ conjugate vaccine following its introduction in Spain. Vaccine 23: 4097-100.

Lasky T, Terracciano GJ, Magder L et al. (1998). The Guillain-Barré syndrome and the 1992-1993 and 1993-1994 influenza vaccines. N Engl J Med 339 : 1797-1802.
Law DKS, Stoltz J, Henderson AM et al (2005). Antigenic and genetic characterization of serogroup C meningococci isolated from invasive meningococcal disease in Canada from 1999 to 2003. Can J Microbiol 51 : 523-30.

Lorange M, Jetté L (2002). Surveillance des infections envahissantes à Neisseria meningitidis. Rapport annuel 2001. Quebec City : Institut national de santé publique, Laboratoire de santé publique du Québec. (http://www.inspq.qc.ca/pdf/ publications/113_Neisseria meningitidis2001.pdf)

Maiden MC, Stuart JM for The U.K. Meningococcal Carriage Group (2002). Carriage of serogroup $\mathrm{C}$ meningococci 1 year after meningococcal C conjugate polysaccharide vaccination. Lancet 359: 1829-30.

MSSS (Ministère de la Santé et des Services sociaux) (2004). Protocole d'immunisation du Québec. Québec (QC) : Ministère de la Santé et des Services sociaux.

National Advisory Committee on Immunisation (2005). Update on meningococcal C conjugate vaccines. CCDR 313 (ACS-3) : 1-4.

National Advisory Committee on Immunisation (NACI) (2007). Statement on conjugate meningococcal vaccine for serogroups A, C, Y and W135. CCDR 33 (ACS-3): 1-24.

Orenstein WA, Bernier R, Hinman AR (1988). Assessing vaccine efficacy in the field. Epidemiol Rev 10: 212-241.

Pichichero M, Casey J, Blatter M et al. (2005). Comparative trial of the safety and immunogenicity of quadrivalent (A, C, Y, W-135) meningococcal polysaccharide-diphtheria conjugate vaccine versus quadrivalent polysaccharide vaccine in two-to tenyear-old children. Pediatr Infect Dis J 24 : 57-62. 
Pichichero ME (2005). Meningococcal conjugate vaccines. Expert Opin Biol Ther 5 : 1475-89.

Pollard AJ (2004). Global epidemiology of meningococcal disease and vaccine efficacy. Pediatr Infect Dis J 23 (supplement) : S274-9.

Pollard AJ, Frasch C (2001). Development of natural immunity to Neisseria meningitidis. Vaccine $19: 1327-46$.

Public Health Agency of Canada (PHAC) (2007). Publicly funded immunization programs in Canada - Routine schedule for infants and children (including special programs and catch-up programs). (http://www.phac-aspc.gc.ca/im/ ptimprog-progimpt/table-1_e.htm)

Ramsay ME, Andrews NJ, Trotter CL et al. (2003). Herd immunity from meningococcal serogroup $C$ conjugate vaccination in England: database analysis. BMJ $326: 365-6$.

Rennels M, King J, Ryall R et al (2002). Dose escalation, safety and immunogenicity study of a tetravalent meningococcal polysaccharide diphtheria conjugate vaccine in toddlers. Pediatr Infect Dis J 21 : 978-979.

Rennels M, King Jr. J, Ryall R et al. (2004). Dosage escalation, safety and immunogenicity study of four dosages of a tetravalent meningococcal polysaccharide diphtheria toxoid conjugate vaccine in infants. Pediatr Infect Dis J 23 : 429-35.

Richmond P, Borrow R, Goldblatt D et al (2001). Ability of 3 different meningococcal $C$ conjugate vaccines to induce immunologic memory after a single dose in UK toddlers. J Infect Dis 183 : 160-3.

Rivest P, Sagot B, Bédard L (1999). Evaluation of the completeness of reporting of invasive meningococcal disease. Can J Public Health 90 : 250-251.

Roscelli JD, Bass JW, Pang L (1991). Guillain-Barré syndrome and influenza vaccination in the US Army, 1980-1988. Am J Epidemiol 133 : 952-5.

Rosenstein NE, Perkins BA, Stephens DS et al. (2001). Meningococcal disease. N Engl J Med $344: 1378-88$.

Sanofi Pasteur Limited (2006). Product monograph. Menactra ${ }^{\mathrm{TM}}$. Meningococcal (groups A, C, Y and W-135) polysaccharide diphtheria toxoid conjugate vaccine. Toronto, Ontario.

Slovic P (1987). Perception of risk. Science 236 : 280-5.

Snape MD, Kelly DF, Salt P et al. (2006). Serogroup $\mathrm{C}$ meningococcal glycoconjugate vaccine in adolescents: Persistance of bactericidal antibodies and kinetics of the immune response to a booster vaccine more than 3 years after immunization. CID 43: 1387-94.

Squires SG, Deeks SL (2004). Enhanced surveillance of invasive meningococcal disease in Canada: 1 January 1999 through 31 December 2001. Can Commun Dis Rep 30 :17-28.

Squires SG, Pelletier L, Mungai M et al. (2000). Invasive meningococcal disease in Canada, 1 January 1997 to 31 December 1998. Can Commun Dis Rep 26-21 : 177-182.

Statistics Canada (1996). Life tables, Canada and Provinces 1990-1992. Catalogue 84-537 Occasional. Ottawa.

Stein KE (1992). Thymus-independent and thymus-dependent responses to polysaccharide antigens. J Infect Dis 165 (Suppl. 1) : S49-52. 
Stephens DS, Greenwood B, Brandtzaeg P (2007). Epidemic meningitis, meningococcaemia, and Neisseria meningitidis. Lancet 369 : 2196-210.

Stratton K, Alamario DA, Wizemann T et al. (2004). Immunization safety review : Influenza vaccines and neurological complications.

Washington, DC : Institute of Medicine.

Trotter CL, Andrews NJ, Kaczmarski EB et al. (2004). Effectiveness of meningococcal serogroup $C$ conjugate vaccine 4 years after introduction. Lancet $364:$ 365-67.

Trotter CL, Edmunds WJ (2002). Modelling cost effectiveness of meningococcal serogroup $C$ conjugate vaccination campaign in England and Wales. BMJ 324 : 1-6.

Trotter CL, Gay NJ, Edmunds WJ (2005). Dynamic models of meningococcal carriage, disease, and the impact of serogroup $C$ conjugate vaccination. Am J Epidemiol $162:$ 89-100.

Tsang RS, Henderson AM, Cameron ML et al (2007). Genetic and antigenic analysis of invasive serogroup Y Neisseria meningitidis isolates collected from 1999 to 2003 in Canada. J Clin Microbiol 45 : 1753-8.
Vu DM, Welsch JA, Zuno-Mitchell P et al. (2006). Antibody persistence 3 years after immunization of adolescents with quadrivalent meningococcal conjugate vaccine. J Infect Dis 193 : 821-8.

Welsh JA, Granoff D (2004). Naturally acquired passive protective activity against Neisseria meningitidis group $C$ in the absence of serum bactericidal activity. Infect. Immun. 72 : 5903-9.

Woo EJ, Ball R, Braun M et al. (2006). Update : Guillain-Barré syndrome among recipients of Menactra ${ }^{\circledR}$ meningococcal conjugate vaccineUnited States, June 2005-September 2006. MMWR 55 (41) : 1120-4.

Zhang Q, Choo S, Everard J et al (2000). Mucosal immune responses to meningococcal Group C conjugate and Group A and C polysaccharide vaccines in adolescents. Infect Immun 68 : 2692-7.

Zhang Q, Pettitt E, Burkinshaw R et al. (2002). Mucosal immune responses to meningococcal conjugate polysaccharide vaccines in infants. Pediatr Infect Dis J 21 : 209-13. 